\title{
Personal technique for definite repair of complete unilateral cleft lip: modified Millard technique
}

Kihwan Han, Jeongseob Park, Seongwon Lee, Woonhyeok Jeong

Department of Plastic and Reconstructive Surgery, Keimyung University Dongsan Medical Center, Keimyung University School of Medicine, Daegu, Korea

\begin{abstract}
Background: Millard's rotation-advancement repair, which is used by many surgeons, can make a natural philtral column, but most surgeons use a modification of the rotationadvancement flap. The purpose of this article is to introduce a modification utilized by the authors and to provide detailed surgical procedure.

Methods: We retrospectively reviewed 82 patients' medical records and presented surgical technique and outcomes. The main features of the authors' strategy are emphasizing horizontal length of the lip, orbicularis oris muscle duplication for improving the definition of the philtral column, overcorrection of domal portion than the non-cleft side in order to compensate for the recurrence during growth. Two judges rated two times the appearance of the patients' nose and lip using Asher-McDade aesthetic index. Intra- and interobserver reliabilities were determined using Cohen's kappa statistics.

Results: All patients recovered eventually after surgery; however, two patients have a minor complications (wound infection in one patient, wound disruption due to trauma in the other patient). The improvement of the aesthetic results can be achieved with this modified Millard technique. Total mean scores of the Asher-McDade index was 2.08, fair to good appearance. The intraobserver reliabilities were substantial to almost perfect agreement and the interobserver reliabilities were moderate to almost perfect agreement.

Conclusion: We modified Millard method for repair of complete unilateral cleft lip. The surgical outcomes were favorable in long-term follow-up. We hope our technique will serve as a guide for those new to the procedure.
\end{abstract}

Keywords: Cleft lip / Rhinoplasty / Millard technique

\section{INTRODUCTION}

Research on cleft lip repair has led surgeons to develop specific techniques for this challenging surgery. In 1955, Millard [1] introduced a rotation-advancement repair, which can make a camouflaged philtral column using a line of closure. This method has the advantage of using maximal available tissue for nasolabial re-

\section{Correspondence: Kihwan Han}

Department of Plastic and Reconstructive Surgery, Keimyung University Dongsan Medical Center, Keimyung University School of Medicine, 56 Dalseong-ro, Jung-gu, Daegu 41931, Korea

E-mail: kihwanhan54@gmail.com

Woonhyeok Jeong

Department of Plastic and Reconstructive Surgery, Keimyung University Dongsan

Medical Center, Keimyung University School of Medicine, 56 Dalseong-ro, Jung-gu,

Daegu 41931, Korea

E-mail: psjeong0918@gmail.com

Received May 8, 2017 / Revised December 30, 2017 / Accepted January 24, 2018 constructions. Since the introduction of this method, many modifications have been developed. A 2005 worldwide survey of leading cleft repair centers found that $84 \%$ of plastic surgeons used variations of the rotation-advancement repair [2]. The authors of the current study have used a modified Millard rotation-advancement technique based on many years of experience. This article introduces a unique complete unilateral cleft lip repair method developed by the senior author (K.H.).

\section{METHODS}

A retrospective medical records review approved by the Keimyung University Dongsan Hospital Institutional Review Board (IRB No. 2017-08-005-001) was conducted on all patients who 
underwent repair of complete unilateral cleft lip between September 1988 and November 2014. Exclusion criteria included patients with a follow-up period shorter than 6 months.

Eighty-two patients included in the study. Besides the frontal view photographs, profile photographs of each patients were assessed, resulting in 164 photographs to be assessed. The mean age at the time of photography was $9.3 \pm 6.2$ years (range, 8 months to 24 years 5 months). The mean age of patients at surgery was $8.3 \pm$ 5.7 months (range, 3.0 to 44.0 months); 46 patients were male and 36 patients were female. The laterality of cleft was 59 left sides and 23 right sides. Patients' characteristics are shown in Table 1.

\section{Surgical technique}

\section{Preoperative design}

The surgeon designed a preoperative marking with a $2.5 \times$ surgical loupe. We used a brilliant green dye for marking within a white line. The landmarks for the preoperative design are shown in Fig. 1. First, we marked the bilateral mouth corners (ch) and a philtral column, which make a connection between the columellar base of the non-cleft side (cphs) and two peaks of Cupid's bow (cphr and cphl). Next, we marked the midpoint of the upper vermilion (ls) and the columellar base of the cleft side (cphl') at the same distance as the ls-cphl. cphl' should not be posed out of the white line with a preservation of white skin roll. The distance of chcphl' could be $2 \mathrm{~mm}$ shorter than the distance of ch-cphr. The length of the medial lip incision was the same length as the philtral column of the non-cleft side. The medial incision line could be drawn using a wire equal to the length of the non-cleft side

Table 1. Summary of patient characteristics

\begin{tabular}{lc} 
Variable & Value \\
\hline No. of patients & 82 \\
Sex & $36(44)$ \\
$\quad$ Female & $46(56)$ \\
Male & \\
The laterality of cleft & $59(72)$ \\
Left & $23(28)$ \\
Right & $8.3 \pm 5.7(3.0-44.0)$ \\
Age at surgery (mo) & $9.3 \pm 6.2(0.7-24.4)$ \\
\hline Age at the time of photography (yr) & \\
\hline Values are presented as number $(\%)$ or mean+standard deviation (range)
\end{tabular}
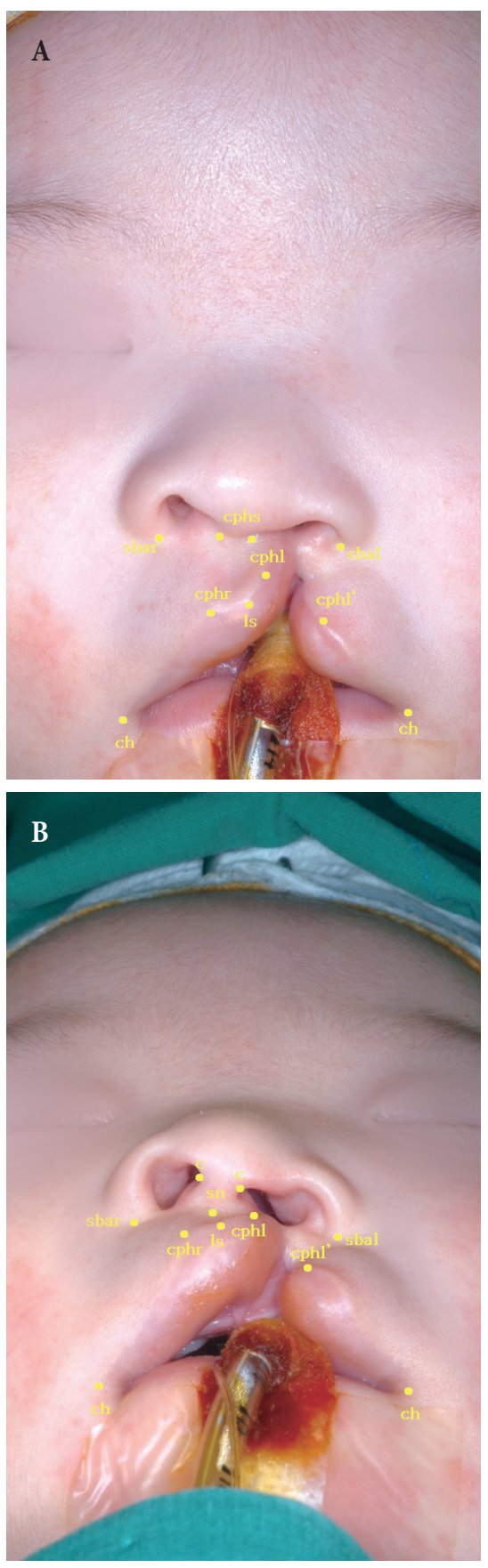

Fig. 1. Anthropometric landmarks for preoperative design. (A) Anterior view, (B) subnasal view. Subalare right and left (sbar and sbal) is the most inferior point of each alar base. The highest point of columella (c) lies atop each hemicolumella and is level with each nostril peak. The crista philtri superior (cphs) is atop each philtral column at the same horizontal line drawn through subnasale (sn). The crista philtri right and left (cphr and cphl) lies at the base of each philtral column (each Cupid's bow peak). The crista philtri left' (cphl') lies at the base of the cleft-side philtral column. The labiale superius (ls) lies at the midpoint of the upper vermilion (Cupid's bow trough), and the cheilion (ch) is located at each labial commissure. 

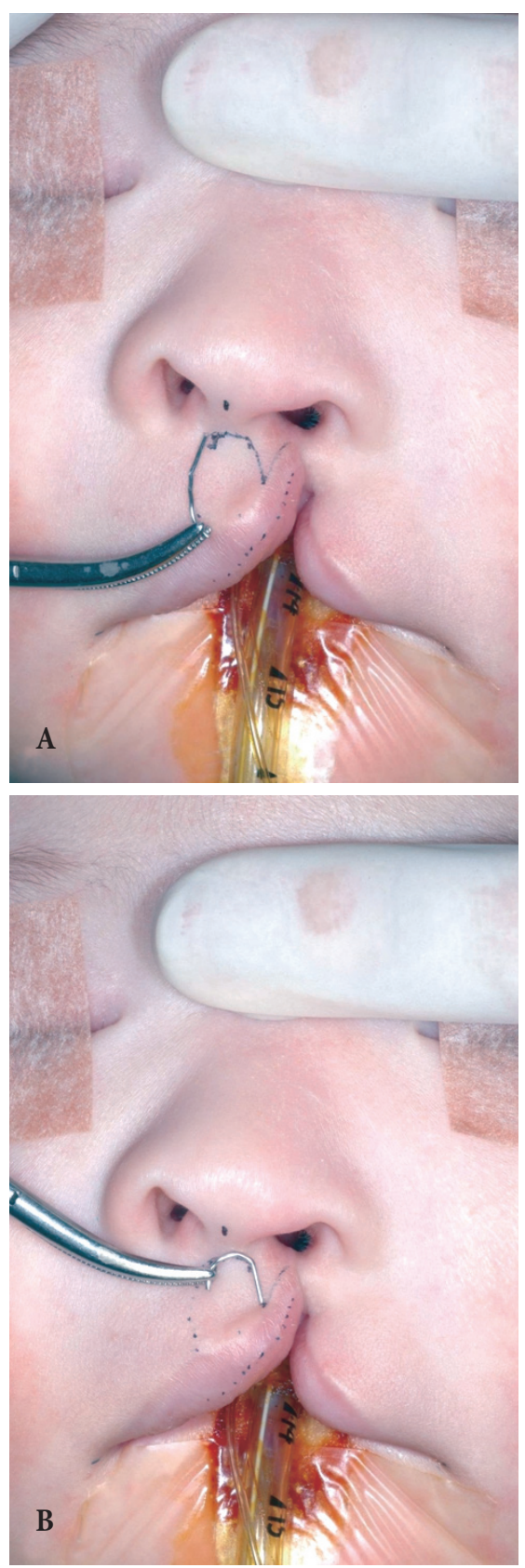

Fig. 2. Surgical design of the medial incision assisted by the guide wire. (A) First, make the guide wire the same length as the non-cleft side philtral column. (B) Mold the guide wire to the same shape as the medial incision to measure whether the rotation is appropriate.

philtral column (Fig. 2). The rotation incision line of the medial lip was drawn in the curve connecting cphl and cphs, horizontal from the columellar-labial junction. This line should not exceed beyond the cphs of the non-cleft side. At this time, a back-cut that is $1 \mathrm{~mm}$ medial to the philtral column of the non-cleft side was drawn. However, the back-cut was restricted in its use because of a wide scar at the columella-labial junction. We drew a line from cphl to the skin-mucosa junction. The C-flap is located between this and the rotation incision.

cphl' of the lateral lip is the most important point in determining the vertical and horizontal lengths of the cleft side lip. Cleft surgeons consider the vertical-lip (sbal-cphl') to be important; if it is short, a noticeable asymmetry can occur. A short horizontal lip is an almost impossible problem to solve and cannot be overlooked. Thus, we try to align the lengths of the vertical lip and the horizontal lip to those of the non-cleft side as much as possible. When we release the abnormally inserted orbicularis oris muscle (OOM) from the dermis, additional lengthening of the vertical height could be obtained. Therefore, we allow a $2 \mathrm{~mm}$ shorter horizontal length of the cleft side compared to that of the noncleft side. The lateral lip incision goes along cphl' to the skin-mucosa junction and is extended to the medial side of the alar base. By not using the upper cut of the advancement flap, we can avoid unacceptable scars (Fig. 3A). We used the laterally based vermilion flap from the lateral lip vermilion to compensate for the thin vermilion of the medial lip (Fig. 3A). The discrepancy between the vermilion height at cphl and cphl' determines the size of the triangular flap. The lateral vermilion incision goes down 1-2 mm vertically from cphl', and the triangular incision is drawn within the vermilion and goes down vertically from the red line. The medial vermilion incision runs down $\sim 1-2 \mathrm{~mm}$ vertically from cphl, and the transverse stab incision is drawn for the advancement of the triangular flap. At this time, careful designs, incisions, and sutures are needed. For the rhinoplasty incision, we use the infracartilaginous incision. The incision of the nasal floor reconstruction draws along the vestibular skin-mucosal junction (Fig. 3B, C).

\section{Definite lip repair}

We tattoo the designs and landmarks using a 30-guage needle so that they are maintained during the operation. We infiltrate the surgical sites using a 1:100,000 mixture of $1 \%$ epinephrine for hemostasis. The medial lip incision runs along the markings using No. 15 blades at the depth of the superficial dermis. Subsequently, 

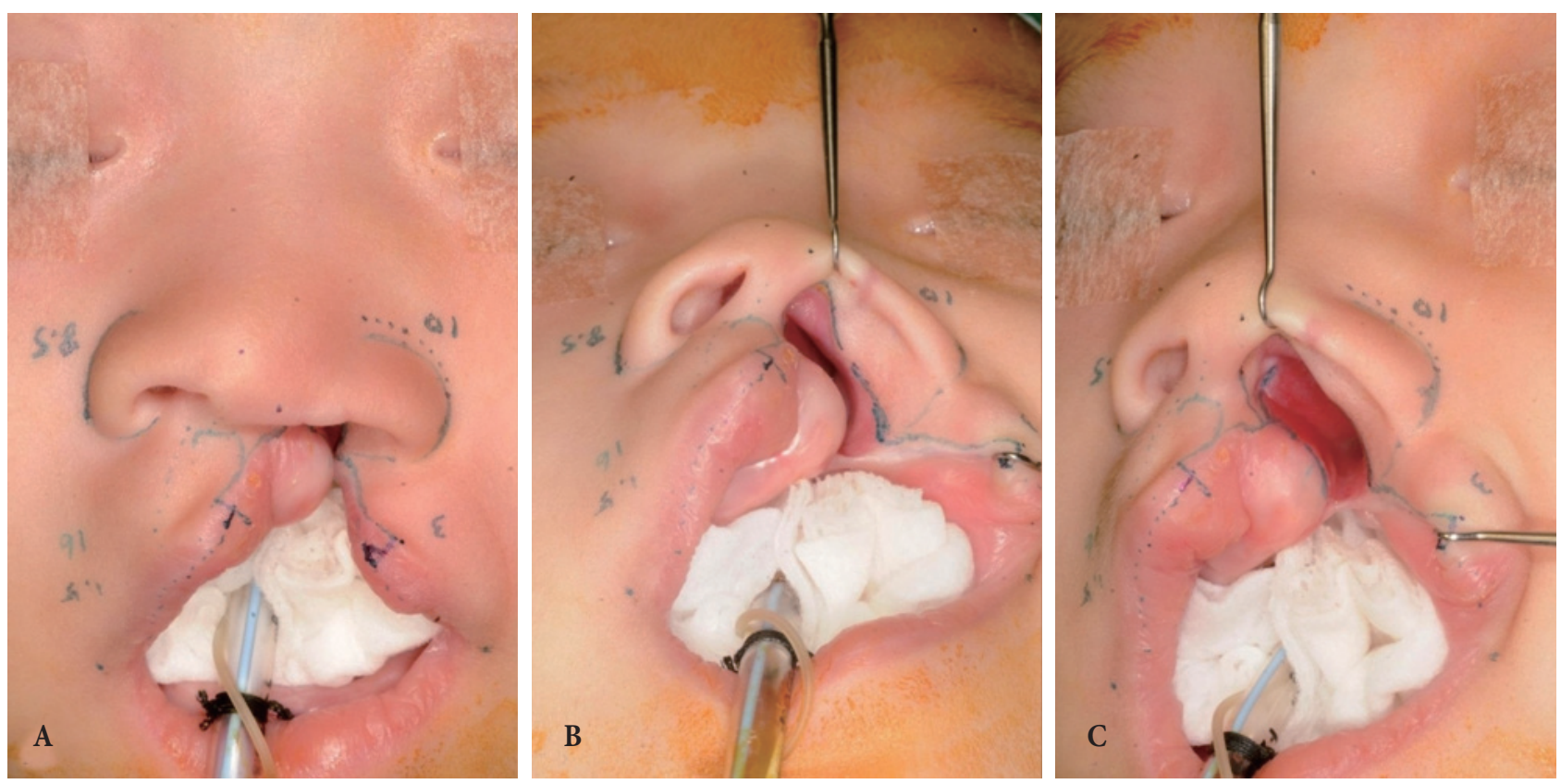

Fig. 3. Final operation design. (A) The back-cut of the medial incision leaves a wide scar; therefore, avoid using this as much as possible. The alar base incision in the lateral lip is also not used. If used, it is limited in the nostril. (B) Worm's eye view. It is possible to confirm the infracartilaginous incision for rhinoplasty and the vestibular skin-mucosa junction for the nasal floor reconstruction. (C) It is possible to confirm the skinmucosa junction of the lateral lip for the nasal floor reconstruction.

we cut full layers, including the muscle layer, using No. 11 blades. The stab incision for inserting the triangular flap of the lateral lip vermilion is cut using No. 15 blades. We dissect the orbicularis oris peripheralis from the medial lip. For making the philtral dimple, we dissect the orbicularis oris peripheralis to slightly over the midline and dissect the orbicularis oris marginalis by only 1 $\mathrm{mm}$. Incision for the nasal floor reconstruction needs careful attention to avoid cutting the septum at the vestibular skin-mucosa junction. We dissect the gingiva along the subperiosteal layer without damaging the tooth follicles using a Freer elevator (Fig. 4).

We score the lateral incision of the lateral lip using No. 15 blades and cut the triangular flap of the vermilion using No. 11 blades. We pull the vermilion mucosa using skin hooks and dissect it using No. 15 blades. The orbicularis oris peripheralis looks like a bulging mass because it has an abnormal dermis insertion at the lateral lip. To dissect the orbicularis oris peripheralis from the dermis, we pull the lateral incision margin using skin hooks, putting inward pressure on the lateral lip with fingers. Then, we dissect 1-2 mm with sharp Metzenbaum scissors and dissect to the melolabial fold (nasolabial fold) with blunt Metzenbaum scissors. We supraperiosteally separate the orbicularis oris peripheralis, which is abnormally inserted to the alar base from the pyriform margin using blunt Metzenbaum scissors (Fig. 4). We then release the alar base from the maxilla sufficiently and dissect from the anterior maxilla to the subperiosteal layer. In this case, we are careful not to damage the infraorbital neurovascular bundle.

\section{Nasal floor reconstruction}

In reconstruction of the nasal floor, we make the nasal lining and oral lining using simple interrupted suture flaps made by an incision on the junction between the bilateral vestibular skin and the mucosa with 5-0 Chromic catgut (Ailee, Busan, Korea).

\section{Primary rhinoplasty}

The lower lateral cartilage of the cleft side is overcorrectively repositioned on the upper lateral cartilage of the cleft side and lower lateral cartilage of the non-cleft side. We approach through the infracartilaginous incision of the cleft side and dissect through the 


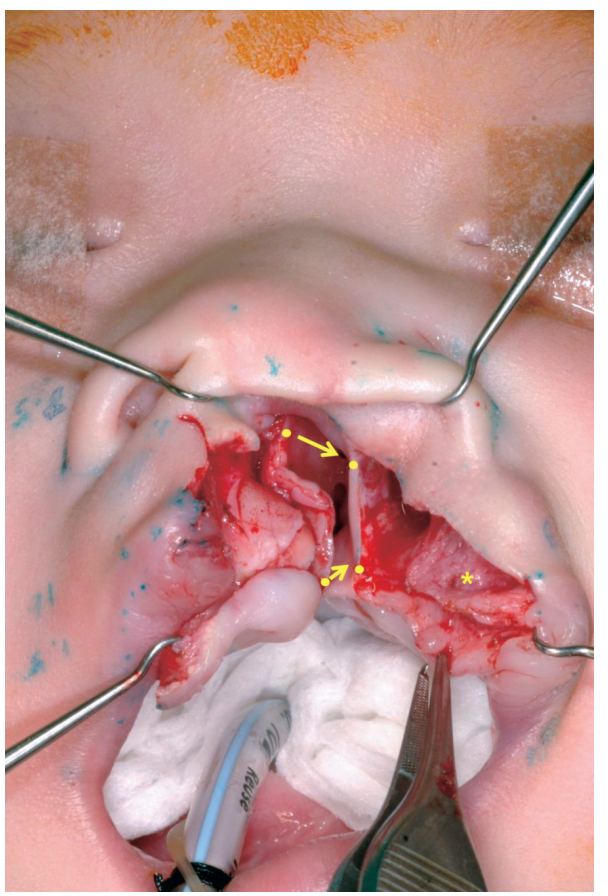

Fig. 4. Nasal floor reconstruction after incision and flap dissection. The oral lining for the nasal floor reconstruction is created by the suturing of the septal mucosa and the vestibular mucosa, as shown in the figure, and the same is true for the nasal lining (arrows). The orbicularis oris peripheralis of the lateral lip is abnormally inserted into the dermis of the lateral lip and alar base (asterisk). It is possible to verify that it has been completely dissected.

supraperichondrial plane. We then locate an anterior nasal spine, confirm the midline, approach between the medial crus and intermediate crus with blunt Metzenbaum scissors, and dissect the lower lateral cartilage through the supraperichondrial plane. We limit the dissection to where the upper lateral cartilage and nasal bone meet through the lower lateral cartilage of the cleft side. We then identify the domal portion of the cleft side lower lateral cartilage, pull back the infracartilaginous incision with a skin hook, put a mosquito forceps in the nostril of the non-cleft side, and press the domal portion to the cleft side. Then, it is possible to confirm the lower lateral cartilage of the non-cleft side. Tattooing the domal portion makes it easy to identify.

We then expose the caudal septum for septal cartilage dislocation. We performed the vertical split incision from the anterior nasal spine and scratch the caudal septum with No. 15 blades to expose it. We dissect the non-cleft side septal mucochondrium along the vomer groove with a Freer elevator. We dislocate the septal cartilage to the cleft side with a Freer elevator and suture it to the anterior nasal spine of the cleft side using 5-0 polydioxanone sutures (PDS).

We apply the interdomal suture and the suture between the upper lateral cartilage of the cleft side and the lateral crus of the lower lateral cartilage to correct vestibular webbing of the lower lateral cartilage. In the interdomal suture, we overcorrect by approximately $3 \mathrm{~mm}$ and suture the domal portion of the non-cleft side tattooed before and the point $3 \mathrm{~mm}$ caudal from the domal portion of the cleft side using 5-0 PDS (Fig. 5). We suture the cephalic border of the lateral crus of the cleft side lower lateral cartilage and the upper lateral cartilage of the same side with 5-0 PDS for suspension. We then apply the inter-foot plate suture using 5-0 PDS and suture the infracartilaginous incision with 5-0 Chromic catgut.

\section{Orbicularis oris muscle overlapping and lip closure}

Orbicularis oris peripheralis muscle overlapping is carried out for the prevention of the cleft side vermilion notch and the formation of the philtral column. We apply the vertical mattress suture to the medial vermilion and the orbicularis oris marginalis of the lateral vermilion for prevention of the cleft side vermilion notch. We apply a $3 \mathrm{~mm}$ overlapped vertical mattress suture for making the philtral column of the cleft side (Fig. 6). First, we pass a 5-0 PDS on the tip of orbicularis oris peripheralis of the medial lip from the bottom. Next, we pass a 5-0 PDS $3 \mathrm{~mm}$ away from the margin of the orbicularis oris peripheralis of the lateral lip from the bottom, and we then pass it from the top to bottom and apply a vertical mattress suture. The cephalic end of orbicularis oris peripheralis is anchored to the septum or the anterior nasal spine for the centralization of Cupid's bow and medialization of the lateral lip.

By inserting a C-flap into the defect of the columellar, which is made by retracting the nostril upward with a Fomon ball retractor, we obtain a sufficient length of columella. We suture the vermilion with 6-0 blue nylon and the cphl-cphl' with 7-0 blue nylon. We apply dermal and skin sutures to the lip skin with 6-0 blue nylon. Then, we suture the dermis and skin of the vermilion flap to fit the red line well using 7-0 blue nylon, trim the excess oral mucosa, and apply a simple interrupted suture with 4-0 Chromic catgut. 

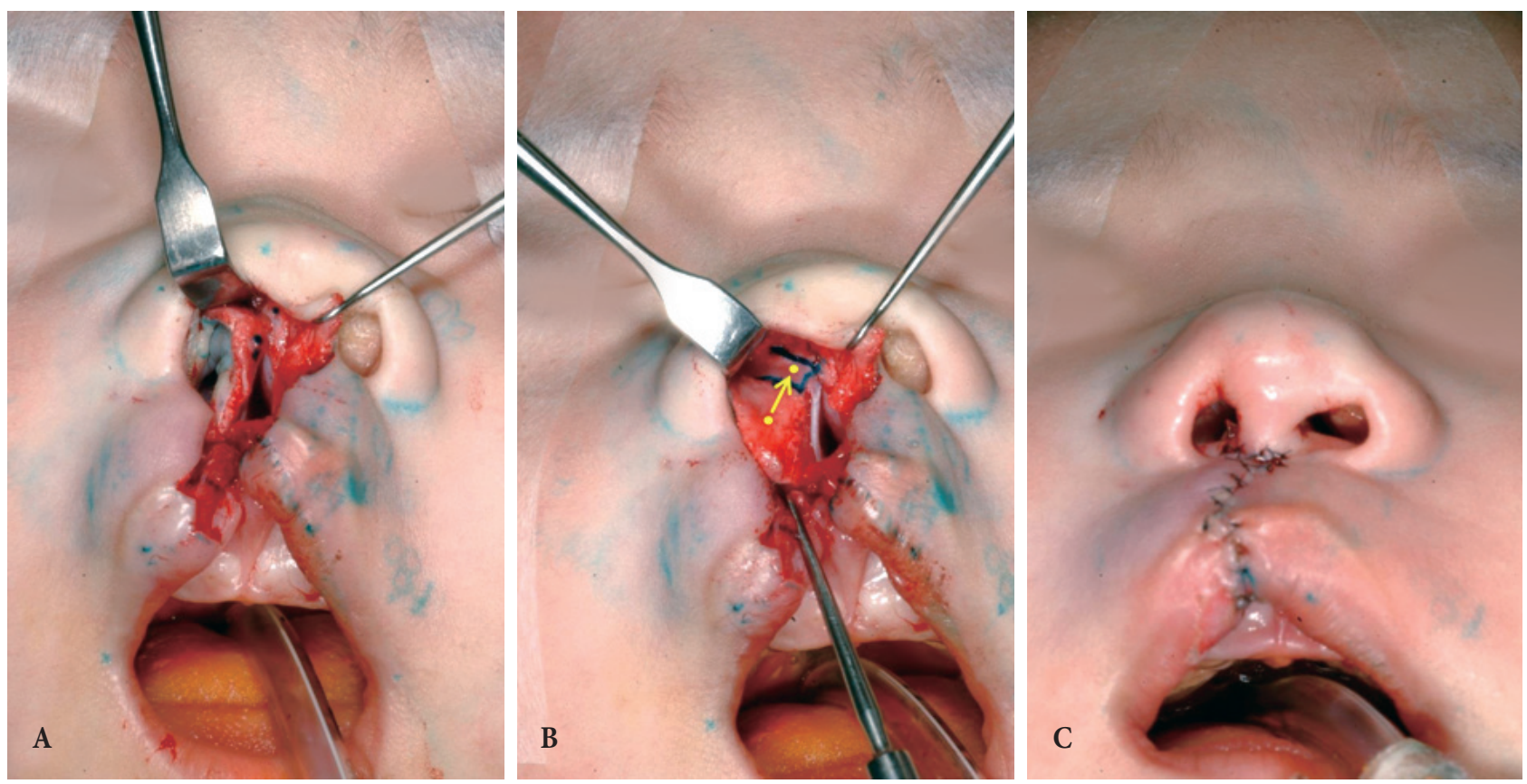

Fig. 5. Primary rhinoplasty. (A) Image shows an overcorrection of a point $3 \mathrm{~mm}$ caudal to the suture to the non-cleft side dome from the cleft side dome. The marked point of the left cleft side is located $3 \mathrm{~mm}$ higher than the point of the non-cleft side. (B) For suturing the upper lateral cartilage (ULC) and lower lateral cartilage (LLC) of the cleft side (arrow), expose the ULC and draw the border of it. Correct the vestibular web by suturing the cephalic margin of the LLC to the ULC. Note that the septal cart is in the upright position. (C) It is possible to confirm that the cleft side nostril is overcorrected compared to the non-cleft side after primary rhinoplasty.

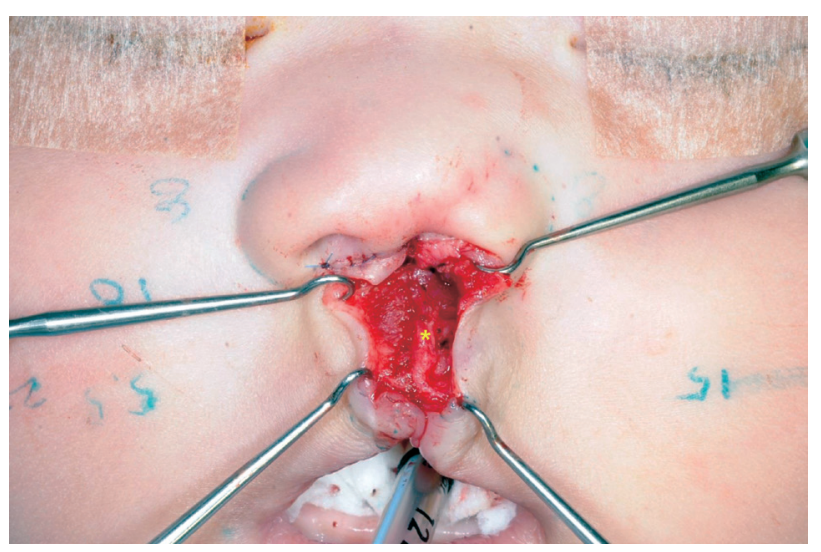

Fig. 6. Overlapping of the orbicularis oris peripheralis. The suture overlaps the lateral lip muscle on the medial lip muscle by $3 \mathrm{~mm}$. The overlapped part (asterisk) improves the definition of philtral column.

\section{Alar transfixion suture}

Finally, we apply the alar transfixion suture (ATS). We overcorrect $1 \mathrm{~mm}$ higher than the non-cleft side alar height and make the stab incisions in the 1 o'clock or 11 o'clock and 2 o'clock or 10 o'clock directions for the site of the ATS in the partial thickness of dermis. The ATS starts from the new alar groove, passes slightly through the superior side of the vestibular web, and then goes back to the stab incision site, ending with ties. After the ATS, it is possible to confirm that the vestibular webbing and blurred alar groove were corrected (Fig. 7).

\section{Postoperative care}

We usually apply an antibiotic ointment to the suture sites, cover the vermilion and lip with padding gauze and compress the vermilion and lip with paper tape (Micropore; 3M, St. Paul, $\mathrm{MN}$, USA). In wide cleft cases, we apply a Logan's bow for decreasing the tension on the open dressing lip suture sites, moisturize the dressing frequently with ointment and cover the nasal tip and ala with paper tape. We remove the stitches in 5 to 7 postoperative days. We apply Steri-Strip (3M) during the 3 months after surgery in order to prevent formation of a wide scar, and the patient wears a nostril conformer for 6 months after surgery. 

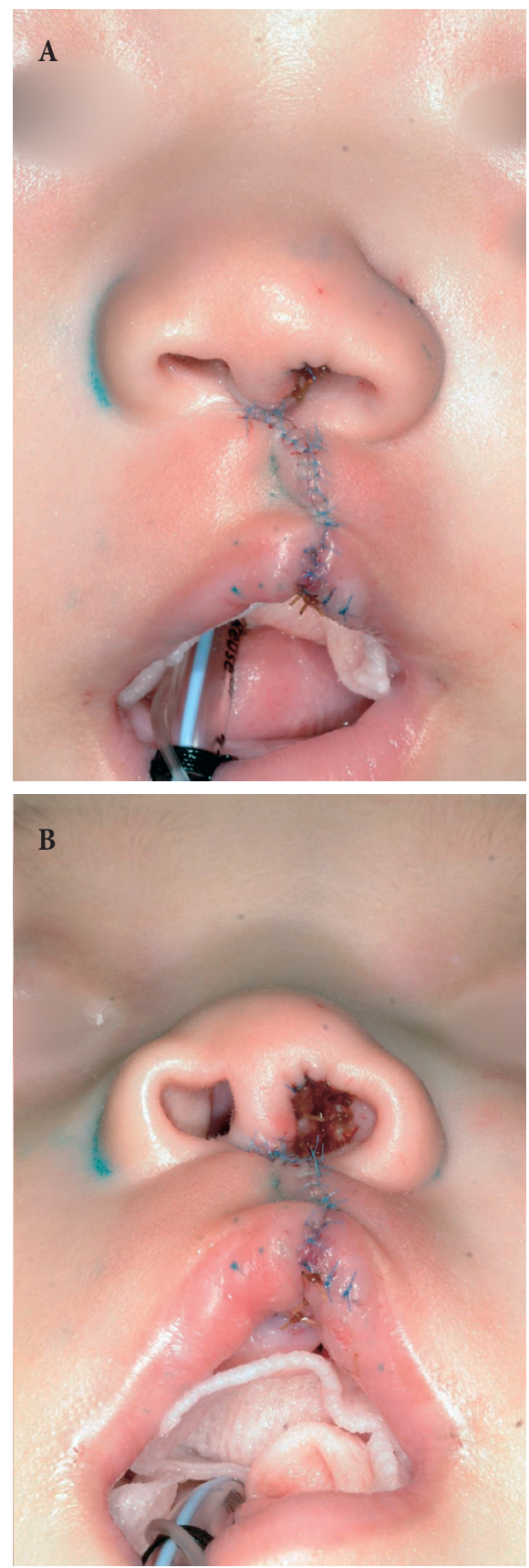

Fig. 7. (A, B) Immediate postoperative photograph.

\section{Analysis of surgical outcomes}

All patients were assessed using the scoring system proposed by Asher-McDade et al. [3]; frequently used system for the assessment of the surgical cleft lip and nose repair [4]. Two plastic surgeons (S. L. and W.J.) rated two times the appearance of the pa- tients' nose and lip using Asher-McDade aesthetic index [3]. Nasal form, deviation of nose, shape of the vermilion border and nasal profile including the upper lip were scored by 5 -point ordinal scale: very good appearance, 1 point; good appearance, 2 points; fair appearance, 3 points; poor appearance, 4 points; very poor appearance, 5 points. Photographs were presented simultaneously as color slide in a PowerPoint presentation in a darkened room. Two weeks after first assessment, the same judges assessed the same number of pictures, once again in random order [5]. The Cohen's kappa statistics was used to measure the degree of agreement between judges (interobserver scores) as well as to determine the intraobserver scores. These figures can be interpreted with reference to Landis and Koch's work (Table 2) [6]. Cohen's kappa statistics was applied using SPSS ver. 18.0 (SPSS Inc., Chicago, IL, USA).

\section{RESULTS}

All patients recovered eventually after surgery; however, two patients have a minor complications; (wound infection in one patient, wound disruption due to trauma in the other patient). The means of the scores was computed for nasal form, nasal deviation, nasal profile and the vermilion border. The means of the total of the four components were also computed. The mean score of the nasal form was 2.51; fair to good appearance. The mean score of the nasal deviation was 1.80; good to very good appearance. The mean score of the vermilion border was 2.24; fair to good appearance. The mean score of the nasal profile was 1.79 , good to very good appearance. And the total mean score was 2.08, fair to good appearance. Favorable aesthetic results were obtained using this modified Millard technique (Fig. 8). The intraobserver reliabilities

Table 2. Interpretation of kappa value [6]

\begin{tabular}{ll} 
Kappa statistics & Strength of agreement \\
$<0.00$ & Poor \\
$0.00-0.20$ & Slight \\
$0.21-0.40$ & Fair \\
$0.41-0.60$ & Moderate \\
$0.61-0.80$ & Substantial \\
\hline $0.81-1.00$ & Almost perfect \\
\hline
\end{tabular}


were substantial to almost perfect agreement and the interobserver reliabilities were moderate to almost perfect agreement. The kappa values for the various comparisons are shown in Tables 3 and 4 . The highest interobserver reliability score was found in the assessment of the shape of the vermilion; substantial to almost perfect agreement (mean, 0.784 ; range, 0.700 to 0.938 ). The lowest
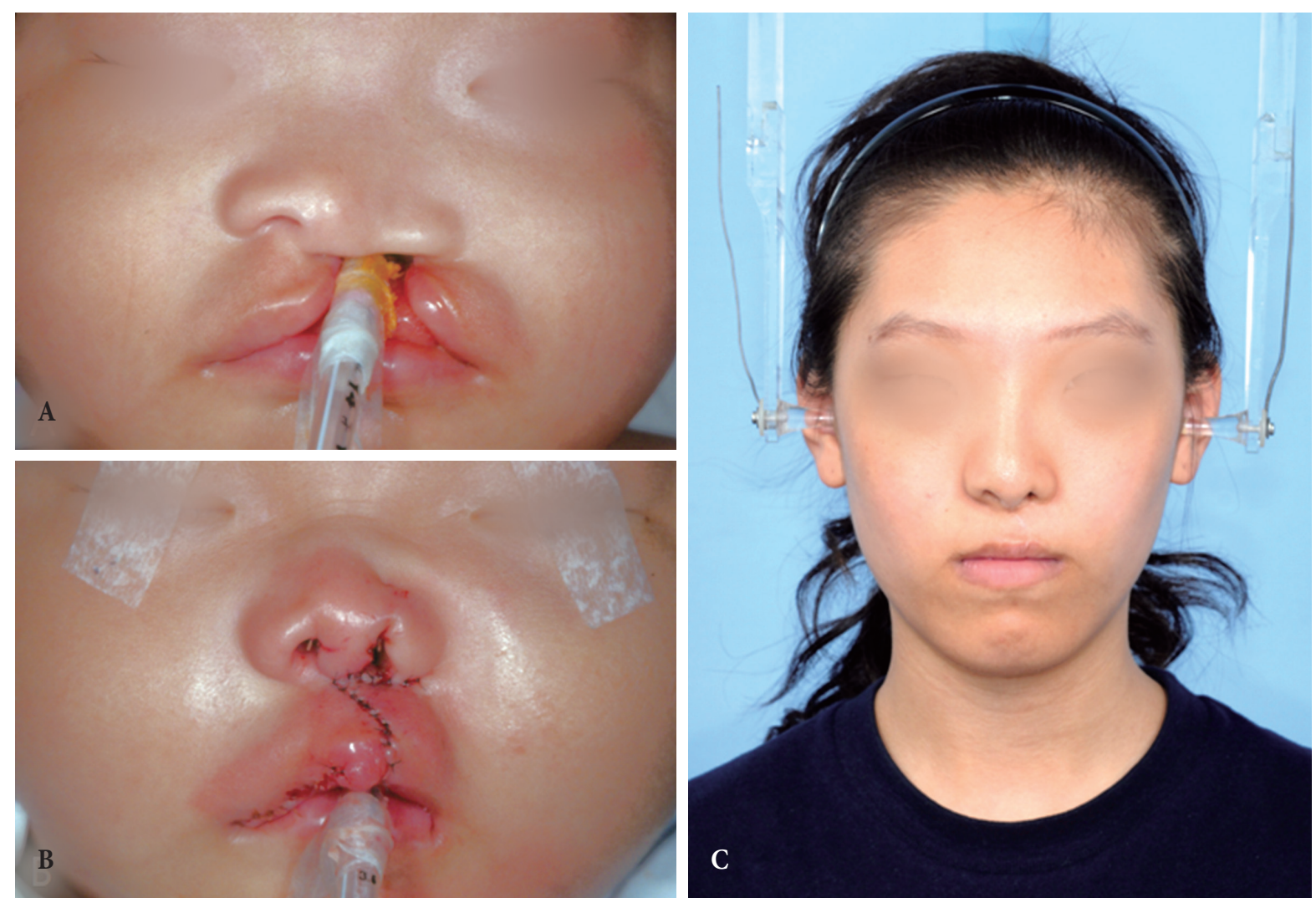

Fig. 8. A girl at 5 months of age at the before of the operation (A), at the end of the operation (B), and at the age of 17 years (C). It would be better to do a nostril rim trimming at primary rhinoplasty.

Table 3. Kappa values for interobserver reliability

\begin{tabular}{lcccc} 
Rater & S.L. 1 vs. W.J. 1 & S.L. 1 vs. W.J. 2 & S.L. 2 vs. W.J. 1 & S.L. 2 vs. W.J. 2 \\
\hline Nasal form & $0.697^{\star \star \star}$ & $0.781^{\star \star \star}$ & $0.578^{\star \star \star}$ & $0.662^{\star \star \star}$ \\
\hline Deviation of nose & $0.562^{\star \star \star}$ & $0.644^{\star \star \star}$ & $0.647^{\star \star \star}$ & $0.683^{\star \star \star}$ \\
\hline Shape of the vermilion border & $0.755^{\star \star \star}$ & $0.938^{\star \star \star}$ & $0.741^{\star \star \star}$ & $0.700^{\star \star \star}$ \\
Nasal profile & $0.751^{\star \star \star}$ & $0.727^{\star \star \star}$ & $0.771^{\star \star \star}$ & $0.632^{\star \star \star}$ \\
\hline
\end{tabular}

1 , first measurement; 2 , second measurement.

${ }^{* * *} p<0.001$.

Table 4. Kappa values for intraobserver reliability

\begin{tabular}{lcc} 
Rater & S.L. 1 vs. S.L. 2 & W.J. 1 vs. W.J. 2 \\
\hline Nasal form & $0.815^{\star \star \star}$ & $0.881^{\star \star \star}$ \\
\hline Deviation of nose & $0.637^{\star \star \star}$ & $0.839^{\star \star \star}$ \\
\hline Shape of the vermilion border & $0.758^{\star \star \star}$ & $0.777^{\star \star \star}$ \\
Nasal profile & $0.863^{\star \star \star}$ & $0.829^{\star \star \star}$ \\
\hline
\end{tabular}

1, first measurement; 2 , second measurement.

${ }^{* * *} p<0.001$. 
interobserver reliability score was found in the assessment of the deviation of nose; moderate to substantial agreement (mean, 0.634 ; range, 0.562 to 0.683 ). The highest intraobserver reliability score was found in the assessment of the nasal form; almost perfect agreement (mean, 0.848 ; range, 0.815 to 0.881 ). The lowest intraobserver reliability score was found in the assessment of deviation of nose; substantial to almost perfect agreement (mean, 0.738 ; range, 0.637 to 0.839 ).

\section{DISCUSSION}

Cleft lip repair has been consistently developed for the functional, cosmetic and psychological-spiritual recovery of the patient. It has been modified countless times since first being described as the simple cutting and approximation of a normal lip. In 1964, Millard [7] reported a modification involving the use of a rotationadvancement flap, and this method has been widely used. The Millard technique has an advantage in that the rotation-advancement flap locates the scar in the philtral column. However, one disadvantage is that this technique makes unacceptable scars around the alar base and leaves a wide scar. To address these disadvantages, Mohler and Fisher's modification was introduced, which modified the rotation-advancement technique of Millard for optimal results [8].

Most cleft surgeons regard the vertical length of the lip as most important and are likely to overlook the horizontal length. Lateralization of the cphl' point is usually performed to extend the vertical length of the lateral lip. We think that it is wrong to excessively emphasize the vertical lengthening because it is almost impossible to correct the horizontal short lip. Generally defined, the cphl' point is at the site where the white roll disappears. Although guarantee of white roll and vertical length is important, the surgeon should be reminded that the horizontal length is also an indispensable point in cleft lip surgery. Therefore, cphl' should be determined, and the surgeon is reminded that an additional 2 $\mathrm{mm}$ of the vertical length and horizontal length could be obtained by subdermal dissection of OOM. While we value horizontal length more than others, we try to preserve the white roll if possible and try to keep the horizontal length and vertical length equal to those of the normal side. Nonetheless, when the vertical length is short, it can be corrected by making a small triangular transposition flap right above the white roll.

In the case of a wide cleft lip, we operate on the adhesion cheiloplasty first, as it is impossible to use the mucosal flap of the lip for the nasal floor reconstruction. We repair the nasal lining reconstruction with some parts of the septal mucosa and the mucosa of the vestibular web, and the oral lining reconstruction with the lip approximation. Our method is inevitably used in patients who underwent the adhesion cheiloplasty, and it is also an easy and effective method for narrow gap cleft lip patients. However, it has been hypothesized that the cartilage septum is relevant to the forward and downward growth. The potential risk of our method is that manipulating the septum and its mucosa is likely to adversely affect the growth of the maxilla. However, a recent investigation reported that the septoplasty with the cleft repair had no effect on the maxillary growth. We feel that there is less maxillary hypoplasia using our method than that seen in previous studies [9].

Primary nasal surgery is closely related to obtaining satisfactory results for cleft lip repair. Although some cleft surgeons disagree with performing primary nasal surgery, the results of primary nasal surgery have been described in many previous studies [10-12]. We conduct primary nasal surgery with the objective of repositioning the displaced lower lateral cartilage and the correction of a deviated septum and the vestibular web. Vestibular webbing comes from the hooding of the lower lateral cartilage caudal margin. This problem can be corrected by an ATS alone, but we correct the issue using the ATS and suture the caudal margins of the lower lateral cartilage and upper lateral cartilage, which then make a cephalic rotation. The ATS acts as a splint, which supports the repositioned lower lateral cartilage and makes the alar groove. The cleft side lower dome of the tip is corrected by the overcorrected interdomal suture. This overcorrection and repositioning of the cleft side dome $3 \mathrm{~mm}$ anterior prepares for the relapse because the cleft side dome is located more posteriorly than the noncleft side dome. The ATS is also used in order to improve the definition of the alar-facial groove and to correct the vestibular webbing.

Previously, we undertook the OOM reconstruction using a 
horizontal mattress suture but experienced a lack of definition of the philtral column due to OOM fibrosis and thinning. More recently, we have found that it is possible to improve the definition of philtral column by superimposing the lateral lip OOM on the top of the medial lip OOM. Variations of this procedure have been introduced by many cleft surgeons [13]. Our method, in contrast to the rotation-advancement flap introduced by Millard, improves the definition of the philtral column by OOM duplication, which complements the depression due to fibrosis during the muscle healing process. Our primary rhinoplasty procedure is different from the conventional method in that the domal portion is more highly overcorrected than the non-cleft side in order to compensate for the recurrence during growth.

The method of assessment we used in this study was a AsherMcDade aesthetic index; one of the most commonly used methods used to measure the aesthetic results of surgical cleft lip and nose repair [5]. When using this scoring system, favorable aesthetic results can be achieved with this modified Millard technique. Especially, our method performed primary nasal surgery, so the result of nasal profile was acceptable. Although OOM duplication was not included in the scale, it would be helpful in defining the philtral column. The reliability has been expressed in the form of kappa values. In this study, the assessment scale showed substantial to almost perfect intraobserver agreement and moderate to almost perfect interobserver agreement. There is a limitation in our study. Although a statistical comparison with the standard method is required, the author did not perform the standard Millard method.

Modern cleft lip repair surgery is not only used for functional recovery but has displayed a growing trend for the precise reconstruction of anatomical geometry. There is no consensus among surgeons for the gold standard procedure, but many cleft surgeons are using the modified Millard technique. Therefore, a basic understanding of this procedure is necessary. We hope that this article helps the reader to understand the rotation-advancement flap technique and serves as a guide for those new to the procedure.

\section{PATIENT CONSENT}

The patients provided written informed consent for the publication and the use of their images.

\section{REFERENCES}

1. Millard DR Jr. A radical rotation in single harelip. Am J Surg 1958;95:318-22.

2. Weinfeld AB, Hollier LH, Spira M, Stal S. International trends in the treatment of cleft lip and palate. Clin Plast Surg 2005;32:19-23.

3. Asher-McDade C, Brattstrom V, Dahl E, McWilliam J, Molsted K, Plint DA, et al. A six-center international study of treatment outcome in patients with clefts of the lip and palate. Part 4: assessment of nasolabial appearance. Cleft Palate Craniofac J 1992;29:409-12.

4. Mosmuller DG, Griot JP, Bijnen CL, Niessen FB. Scoring systems of cleft-related facial deformities: a review of literature. Cleft Palate Craniofac J 2013;50:286-96.

5. Mosmuller DG, Bijnen CL, Kramer GJ, Disse MA, Prahl C, Kuik DJ, et al. The Asher-McDade aesthetic index in comparison with two scoring systems in nonsyndromic complete unilateral cleft lip and palate patients. J Craniofac Surg 2015;26:1242-5.

6. Landis JR, Koch GG. The measurement of observer agreement for categorical data. Biometrics 1977;33:159-74.

7. Millard DR Jr. Rotation-advancement principle in cleft lip closure. Cleft Palate J 1964;12:246-52.

8. Vyas RM, Warren SM. Unilateral cleft lip repair. Clin Plast Surg 2014;41:165-77.

9. Janiszewska-Olszowska J, Gawrych E, Wedrychowska-Szulc B, Stepien P, Konury J, Wilk G. Effect of primary correction of nasal septal deformity in complete unilateral cleft lip and palate on the craniofacial morphology. J Craniomaxillofac Surg 2013;41:468-72.

10. Anderl H, Huss $H$, Ninkovic M. Primary simultaneous lip and nose repair in the unilateral cleft lip and palate. Plast Reconstr Surg 2008;121:959-70.

11. Chang CS, Por YC, Liou EJ, Chang CJ, Chen PK, Noordhoff MS. Long-term comparison of four techniques for obtaining nasal symmetry in unilateral complete cleft lip patients: a single surgeon's experience. Plast Reconstr Surg 2010;126:1276-84.

12. Mathes SJ, Hentz VR. Plastic surgery. Vol. 3. the head and neck: part 2. Philadelphia: Saunders; 2006.

13. Grotting JC, Neligan PC. Plastic surgery. Vol. 3. craniofacial, head and neck surgery and pediatric plastic surgery. Philadelphia: Saunders; 2013.

\section{CONFLICT OF INTEREST}

No potential conflict of interest relevant to this article was reported. 